ISSN: 2600-5859

\title{
Simulación de esfuerzos mecánicos sobre las férulas para miembros superiores
}

\section{Simulation of mechanical stresses on ferule for upper limbs}

Edwin Rodolfo Pozo Safla. ${ }^{1}$, Sócrates Miguel Aquino Arroba. ${ }^{2}$, Geovanny Guillermo Novillo Andrade. ${ }^{3} \&$ Edwin Andres Castelo Guevara. ${ }^{4}$

Abstract.

DOI: https://doi.org/10.33262/concienciadigital.v3i1.2.1202

The supported computer simulation of the CAE software helps to validate the prototypes, which have features of functionality that is to say that they are subject to operational loads, the simulation is based on the application of the preprocess, process and postprocess stages. In the preprocessing stage the geometric conditions of the splints are determined, which are obtained through the application of reverse engineering such as 3D scanning, in this stage the type of material with which the splint will be constructed for this PLA (Polylactic Acid) material research is proposed, the boundary conditions are established that are the operating restrictions as well as the loads that will be submitted, an analysis of the mesh quality is established so that the results converge.

Keywords: CAE, PLA, ABS, Ferule

${ }^{1}$ Escuela Superior Politécnica de Chimborazo, Facultad de Mecánica. Riobamba, Ecuador, edwin.pozo@espoch.edu.ec

${ }^{2}$ Escuela Superior Politécnica de Chimborazo, Facultad de Mecánica. Riobamba, Ecuador, saquino@espoch.edu.ec

${ }^{3}$ Escuela Superior Politécnica de Chimborazo, Facultad de Mecánica. Riobamba, Ecuador, gnovillo@espoch.edu.ec

${ }^{4}$ Riobamba, Ecuador, andcas942@gmail.com 


\section{Resumen.}

La simulación computacional apoyada del software CAE ayuda a validar los prototipos, los mismos que tienen características de funcionalidad es decir que están sometidas a cargas operativas, la simulación se basa en la aplicación de las etapas de preproceso, proceso y postproceso. En la etapa del preproceso se determina las condiciones geométricas de las férulas las mismas que son obtenidas mediante la aplicación de la ingeniería inversa como es el escaneo en $3 \mathrm{D}$, en esta etapa se definirá el tipo de material con la que se construirá la férula para esta investigación se propone el material PLA (Polylactic Acid), se establece las condiciones de contorno que son las restricciones de funcionamiento al igual que las cargas que serán sometidas, se establece una análisis de la calidad de malla para que los resultados lleguen a converger. En la etapa de proceso se establecen parámetros de resolución en función de los resultados que se requieren obtener análisis mecánico estructural junto con una herramienta de optimización topológica. En la etapa de postproceso, se estable los resultados de deformación y esfuerzos que son producidos por la acción de las cargas actuantes en la férula, donde se puede establecer algunas imperfecciones sobre la superficie de la férula, con la información proporcionada de análisis CAE, se llega a determinar su correcto funcionamiento y resistencia mecánica para la etapa final que es la manufactura.

Palabras claves: CAE, PLA, ABS, Férula

\section{Introducción.}

Los miembros superiores representan para las personas una herramienta funcional y actuante que involucra el uso de fuerza para sujetar elementos, habilidades de coordinación y movimientos para el desarrollo de múltiples actividades diarias.

\section{Ortesis de miembros superiores}

Las ortesis son elementos biomecánicos aplicados externamente con el propósito de restaurar o mejorar la funcionalidad del sistema músculo esquelético. En general los problemas músculo esqueléticos incluyen aquellos relacionados al trauma, deporte e injurias relacionadas al trabajo (accidentes laborales). Las ortesis de miembros superiores también se utilizan frecuentemente en pacientes con problemas neurológicos, tales como un Evento Vascular Cerebral, traumatismo encefalocraneal, parálisis cerebral, lesiones medulares y de nervios periféricos. De igual manera, en condiciones artríticas (AR), los materiales utilizados para su fabricación son los termoplásticos de baja temperatura (orthoplast, aquaplast ${ }^{\circledR}$, entre otros) o de alta temperatura (polivinílico-PVC, acrílico, plexidur), que pueden ser prefabricados o confeccionados a medida. Otros materiales incluyen, yeso, metal, elásticos y velcro®. Estos elementos pueden ser confeccionados por el Terapeuta Ocupacional, el Fisioterapeuta (especializado en lesiones de mano) y el Ortesista. (Arce, 2005) 
ISSN: 2600-5859

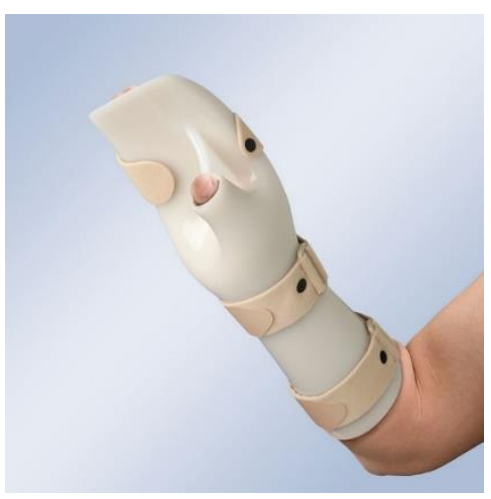

Figura. 1. Ortesis de miembro superior

\section{Ortesis antebrazo-muñeca-mano}

Su ubicación es palmar (volar), dorsal o circunferencial y puede aplicarse desde la punta de los dedos hasta los $2 / 3$ del antebrazo. La muñeca está en posición neutral o en ligera flexión dorsal. Las indicaciones de este tipo de ortesis incluyen las siguientes: (Arce, 2005)

- Inmovilización en pacientes con tendinitis (flexor o extensor), o en aquellos sometidos a reparación tendinosa, nerviosa o fracturaría.

- Mantenimiento de ROM pasivo en pacientes con lesiones de motoneurona superior (MNS), quemaduras y contracturas.

- Los tipos más específicos de estas ortesis incluyen:

- Ortesis de reposo funcional (functional resting splint)

- Ortesis estática de mano (static hand splint)

- Ortesis para quemados (burn splint)

- Ortesis de descarga de peso (weight-bearing splint)

- Ortesis de reposo de mano (resting hand splint)

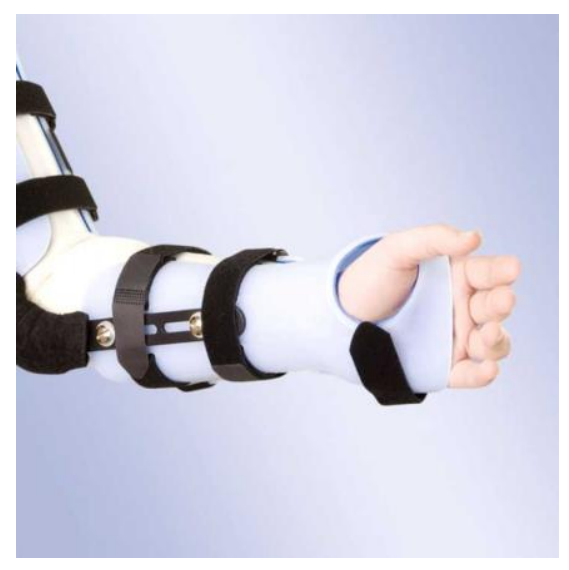

Figura. 2. Ortesis antebrazo-muñeca-mano 
En la Figura 3 se indica el concepto de diseño de un prototipo, donde se realiza primero la forma del modelo sólido con la utilización de herramientas CAD que es el dibujo asistido por computadora. El prototipo inicial casi siempre se establece en función de la experiencia del diseñador, se puede utilizar los datos heredados de prototipos anteriores o de la obtención mediante ingeniería inversa. El diseño de la férula se define dominios específicos para ayudar a conseguir la personalización del modelo y encontrar una solución a un problema de rehabilitación de miembro superior, el modelo virtual se respalda del diseño conceptual tomada del estado del arte. Además, con un sistema integrado de la herramienta CAD puede generar directamente un modelo sólido del diseño conceptual que sirva exclusivamente para las simulaciones de diseño y fabricación.

Con el prototipo de la férula representado en $\mathrm{CAD}$, se pueden realizar simulaciones para el rendimiento, la confiabilidad y la fabricación de la férula. Elaborado de un modelo de producto de diseño asistido por computadora centralizado, los modelos de simulación pueden derivarse con simplificaciones y suposiciones adecuadas.

Hay que considerar, un análisis unidireccional que rige los cambios de modelos CAD a modelos de simulación debe ser establecido para actualizaciones rápidas de modelos de simulación. El análisis mantiene la coherencia entre los modelos de simulación y CAD durante todo el ciclo de desarrollo de la férula. (Chang, 2014)

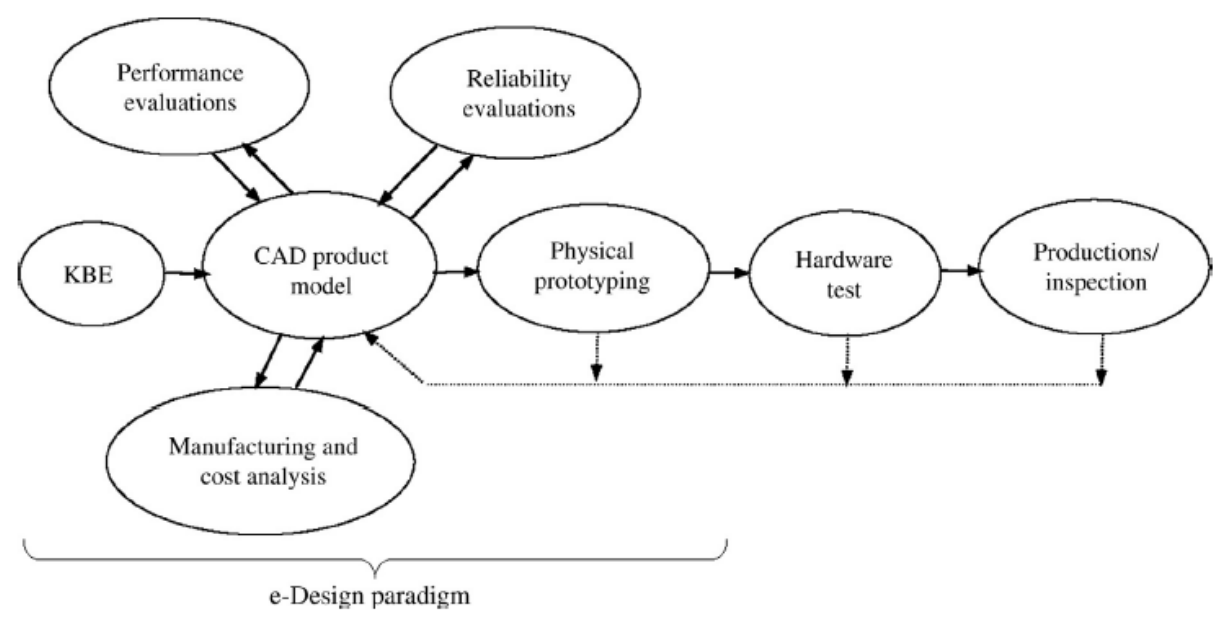

Figura. 3. El paradigma del diseño

El desempeño, la calidad y los costos obtenidos de las simulaciones multifisicas son variables de diseño incluyendo dimensiones geométricas y el material. 
Con el modelo CAD de la férula, se puede realizar un enfoque de diseño sistemático, que incluye un estudio paramétrico para el diseño conceptual y un estudio de compensación para el diseño de detalles, para mejorar la férula con un número mínimo de iteraciones de diseño.

La férula diseñada en el entorno virtual se construye utilizando equipos de creación rápida de prototipos como impresión 3D. Los prototipos físicos son compatibles mediante la verificación del diseño mediante elementos finitos y la verificación del ensamblaje a nivel de tolerancias dimensionales y geométricas.

El análisis de elementos finitos (FEA), se basa en la idea de construir un objeto complicado con bloques simples, o dividir un objeto complicado en piezas más pequeñas y manejables. La aplicación de esta simple idea se puede encontrar en cualquier lugar de la vida cotidiana. El FEA se puede aplicar para resolver los modelos matemáticos de muchos problemas de ingeniería, desde el análisis de tensión de estructuras de armazones y armazones o máquinas complicadas, hasta respuestas dinámicas bajo diferentes mecanismos.

Un esbozo del proceso de desarrollo de productos asistido por ordenador. (Chen, 2005)

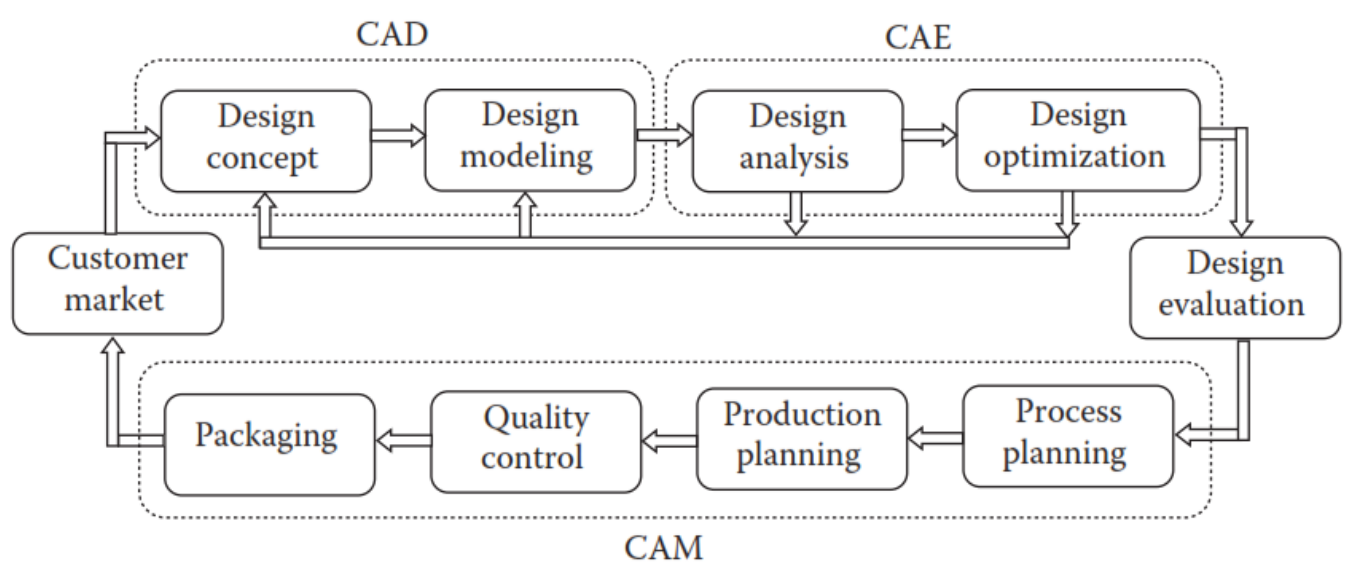

Figura. 4. Desarrollo de productos asistidos por computador

En las últimas décadas, muchos programas comerciales han estado disponibles para realizar la FEA. Entre una amplia gama de soluciones de simulación de elementos finitos provistas por las principales compañías de CAE, ANSYS® Workbench es una plataforma fácil de usar diseñada para integrar a la perfección la tecnología. Ofrece conexión bidireccional a los principales sistemas CAD. El entorno Workbench está orientado a mejorar la productividad y la facilidad de uso entre los equipos de ingeniería. Ha mejorado como una herramienta indispensable para el desarrollo de productos en un número creciente de empresas y centros de investigación, encontrando aplicaciones en diversos campos de la ingeniería. 
Las propiedades mecánicas del ácido láctico o su nombre comercial (PLA) pueden variar, desde materiales suaves y elásticos hasta materiales rígidos y de alta resistencia, de acuerdo con diferentes parámetros, tales como cristalinidad, estructura del polímero, peso molecular y formulación del material (mezclas, plastificantes, compuestos, etc.) y orientación. (Sin, 2012)

La Tabla 1 resume algunas de las propiedades mecánicas de PLA desarrolladas por NatureWorks LLC.

\begin{tabular}{|c|c|c|c|c|c|c|}
\hline Properties* & $\begin{array}{l}\text { Ingeo }^{\mathrm{TM}} \\
\text { 2003D }\end{array}$ & $\begin{array}{l}\text { ASTM } \\
\text { Method }\end{array}$ & Ingeo $^{\mathrm{TM}} 3801 \mathrm{X}$ & $\begin{array}{l}\text { ASTM } \\
\text { Method }\end{array}$ & $\begin{array}{l}\text { Ingeo }^{\mathrm{TM}} \\
\text { 8052D }\end{array}$ & $\begin{array}{l}\text { ASTM } \\
\text { Method }\end{array}$ \\
\hline Tensile strength, MPa (psi) & $53(7700)$ & D882 & - & - & - & - \\
\hline Yield strength, MPa (psi) & $60(8700)$ & D882 & $25.9(3750)$ & D638 & $48(7000)$ & D638 \\
\hline $\begin{array}{l}\text { Young's modulus, GPa } \\
\text { (kpsi) }\end{array}$ & $3.5(500)$ & D882 & $2.9(432)$ & D638 & - & D638 \\
\hline Elongation at break, \% & 6.0 & D882 & 8.1 & D638 & 2.5 & D638 \\
\hline $\begin{array}{l}\text { Notched Izod Impact, J/m } \\
\text { (Ib.ft/in) }\end{array}$ & $12.81(0.24)$ & D256 & $144(2.7)$ & D256 & $16(0.3)$ & D256 \\
\hline Flexural strength, MPa (psi) & - & - & $44(6400)$ & D790 & $83(12,000)$ & D790 \\
\hline $\begin{array}{l}\text { Flexural modulus, GPa } \\
\quad(\mathrm{kpsi})\end{array}$ & - & - & $2.85(413)$ & D790 & $3.8(555)$ & D790 \\
\hline
\end{tabular}

Tabla. 1. Propiedades mecánicas del PLA

Las propiedades mecánicas del PLA puro pueden variarse cambiando la estereoquímica, la cristalinidad, el peso molecular, etc. El PLA con alta pureza estereoquímica posee la característica de alta resistencia a la tracción y módulo, pero carece de resistencia al impacto. En contraste, el copolímero de L-lactida y D-lactida permanece en un estado amorfo, que tiene propiedades mecánicas pobres. Los investigadores tienden a utilizar la técnica de copolimerización para modificar las propiedades existentes de PLA, con el fin de ampliar sus aplicaciones. Además, se han utilizado técnicas de mezcla de polímeros para combinar las propiedades del PLA con las de otro polímero para lograr un mejor impacto y resistencia a la flexión. En general, la mayoría de las modificaciones hechas a PLA están dirigidas a mejorar sus propiedades mecánicas mientras mantienen su biodegradabilidad.

El desarrollo de PLA, permite que la mayoría de sus aplicaciones están en el campo biomédico. Es ampliamente utilizado para proporcionar soporte estructural temporal para la unión y el crecimiento de tejidos en cirugía. También se utiliza como portador de fármacos, que contiene agentes activos de liberación controlada para tratamientos a largo plazo, incluso para el cáncer.

\section{Metodología}

\section{Obtención del prototipo virtual de la férula}

La obtención del modelo virtual para la modelación de la férula se hace con uso del escáner 3D Go!SCAN 50 y la interface del software Vx-element, se prepara el miembro superior de la persona para escanear ubicando puntos para el reconocimiento del láser, creando una nube de putos en el 
espacio $(\mathrm{x}, \mathrm{y}, \mathrm{z})$ en un formato *.cst y luego se puede exportar como *.stl binario, como se puede observar en la figura 5.
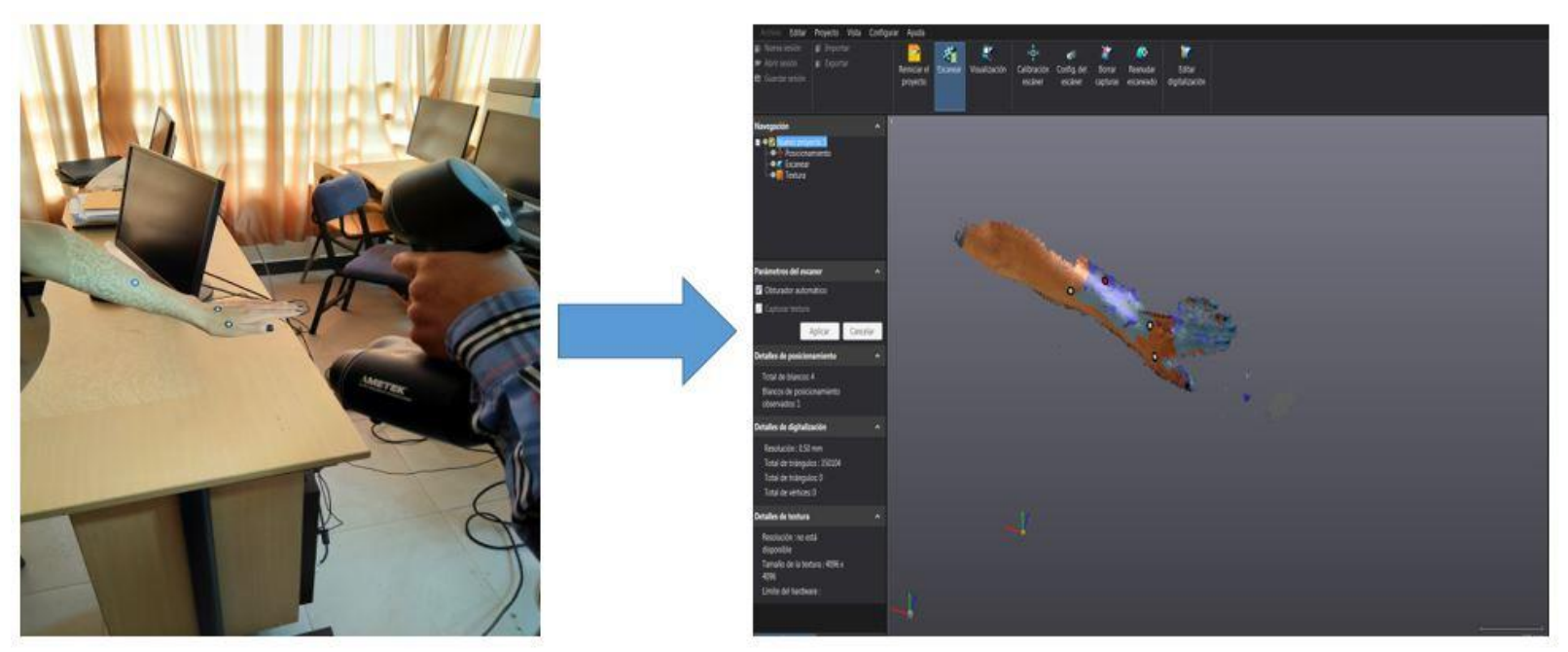

Figura. 5. Preparación y Escaneado del miembro superior.

El proceso de escaneo genera una nube de puntos para editar con el software Geomagic Design que permite transformar con las herramientas a un modelo solido que sirva de molde para el diseño de la férula.
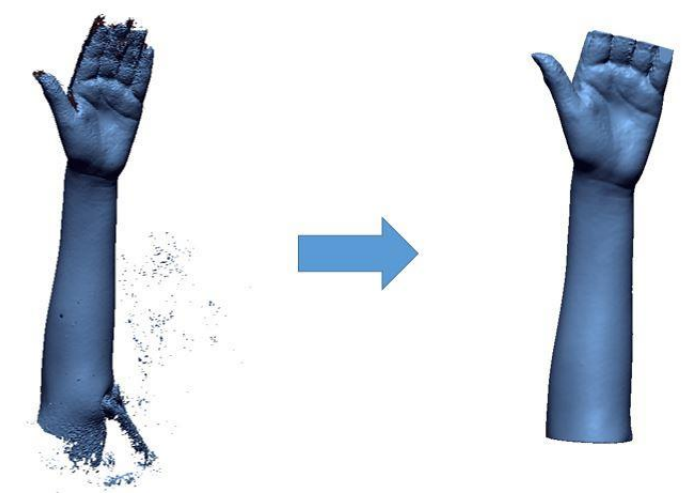

Figura 6 Trasformación de la nube de puntos a sólido.

Una vez realizado el modelo tridimensional con la ayuda de herramientas de croquizado, operaciones de superficie y extrusiones se evalúa la calidad de la formal final con la nube de puntos como referencia, diagnosticando las superficies que tienen una desviación geométrica y que se identifica a través de una cinta de colores, de acuerdo a la escala el color verde identifica que no 
ISSN: 2600-5859

existe ninguna desviación, el azul muestra un valor menor al espesor especificado y el extremo rojo el valor mayor al espesor como se muestra en la figura 7.
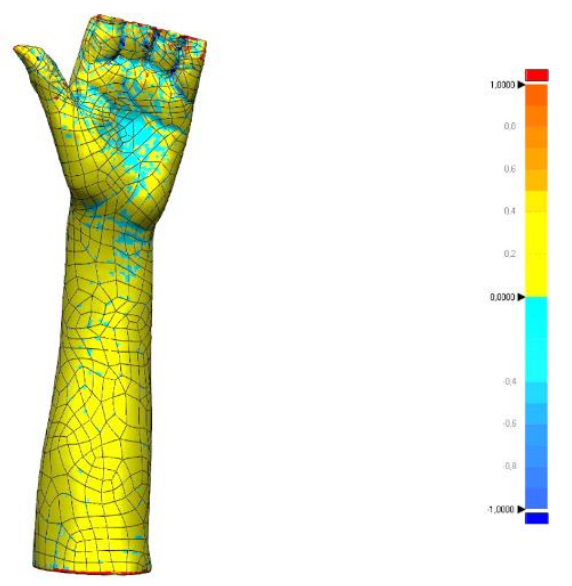

Figura 7. Evaluación de la Geometría de miembro superior.

Finalmente, rectificado el sólido se exporta a un software especializado en el CAD, para generar el modelo de la férula personalizada la misma que es construida con operaciones de superficies tomando como molde el modelo escaneado-transformada a sólido. Y se genera un modelo de férula inicial quien será sometido a esfuerzos y se valorará su comportamiento.

Se genera el modelo 3D de la férula con algunos detalles del personalizado como se muestra la figura 8 .

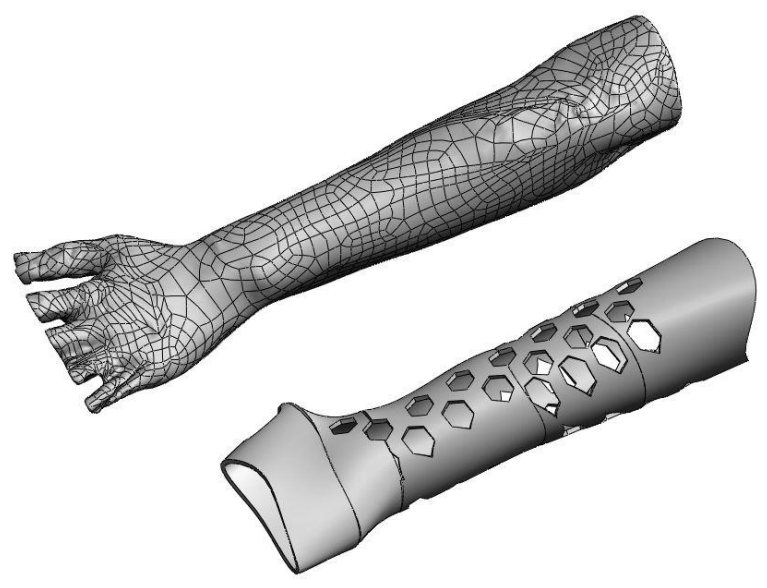

Figura. 8. Modelación de la férula.

Definición del problema. 
ISSN: 2600-5859

El elemento CAD para realizar la simulación que es la férula se obtuvo por medio de la aplicación de herramientas de ingeniería inversa, por lo tanto, es importante tener la geometría totalmente definida, en la figura 9 se muestra la férula para el análisis, el mismo que contiene el material adecuado, restricciones y condiciones de carga a las que está sometido para encontrar valores de deformación estructural y esfuerzos mecánicos.
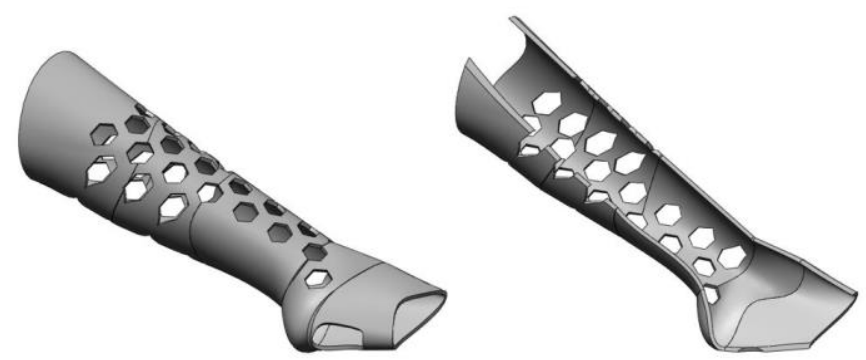

Figura. 9. Férula para el análisis.

Pre proceso

Definición de propiedades mecánicas del material

La figura 10 muestra las consideraciones de las propiedades mecánicas del polímero PLA, con las que se realiza la simulación en el software; utilizando el método de elemento finito. (Molina Osejos, 2016)

\begin{tabular}{|c|c|c|}
\hline Property & Value & Unit \\
\hline 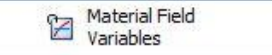 & 贯 Table & \\
\hline Density & 1.24 & $\mathrm{~kg} \mathrm{~m} \mathrm{~m}^{\wedge}-3$ \\
\hline 曰 7 Isotropic Elasticity & & \\
\hline Derive from & Young's Modulus an... $=$ & \\
\hline Young's Modulus & $3.5 E+09$ & $\mathrm{~Pa}$ \\
\hline Poisson's Ratio & 0.39 & \\
\hline Bulk Modulus & $5.303 E+09$ & $\mathrm{~Pa}$ \\
\hline Shear Modulus & $1.259 \mathrm{E}+09$ & $\mathrm{~Pa}$ \\
\hline 7 Tensile Yield Strength & 50 & $\mathrm{~Pa}$ \\
\hline$\bigoplus \begin{array}{l}\text { Compressive Yield } \\
\text { Strength }\end{array}$ & 80 & $\mathrm{~Pa}$ \\
\hline $\begin{array}{l}\text { Tensile Ultimate } \\
\text { Strength }\end{array}$ & 60 & $\mathrm{~Pa}$ \\
\hline
\end{tabular}

Figura 10. Propiedades mecánicas del PLA

Definición de calidad de la Malla

Según la aplicación del método de elemento finito se establece la discretización del medio físico o continuo para conseguir un número finito de pequeños tetraedros de control. Con el objetivo de observar la influencia de la discretización se generan varios modelos con distintos tipos de mallado entre ellos hexaedros que permita construir un modelo de malla con buena calidad y así obtener buenos resultados (Lee, 2018) 


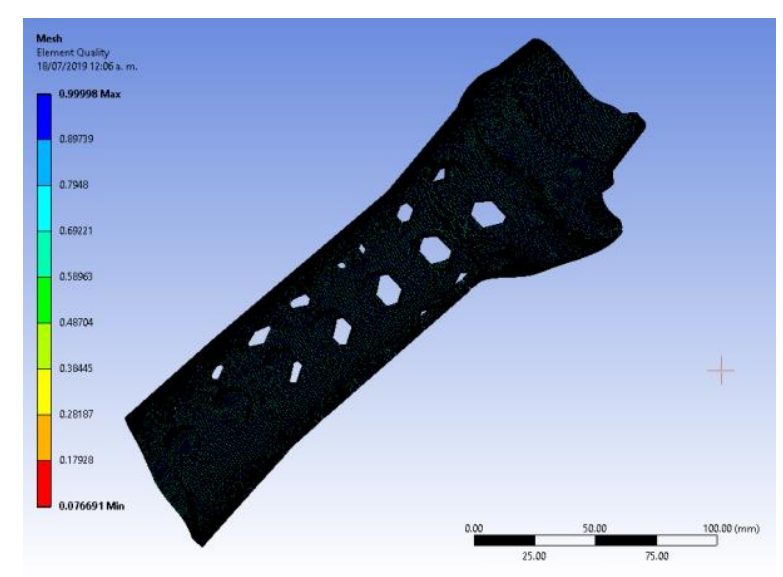

Figura. 11. Malla con elementos tetraédricos.

\section{Definición del modelo de férula para simulación}

Los diagramas de cuerpo libre (DCL) se grafican para ayudar a identificar las fuerzas y los momentos que actúan sobre partes individuales de un sistema y para aplicar el uso correcto de las ecuaciones de la mecánica y para analizar el problema. Para este objetivo, los elementos que constituyen un sistema están aisladas de su entorno y los efectos del entorno son reemplazados por fuerzas y momentos apropiados. (Nordin \& Frankel, 2001)

En algunos estudios, el planteamiento puede ser investigar las fuerzas involucradas en la férula y alrededor de varias articulaciones del cuerpo humano para diferentes condiciones posturales y de carga.

Estos análisis pueden realizarse separando el cuerpo en dos partes en la articulación de interés y dibujando el diagrama de cuerpo libre de una de las partes. Se considera a una persona en un dispositivo de ejercicio que sostiene un asa que está conectada a un cable como se muestra en la figura 12. La condición postural ayuda a determinar la interacción de las fuerzas.

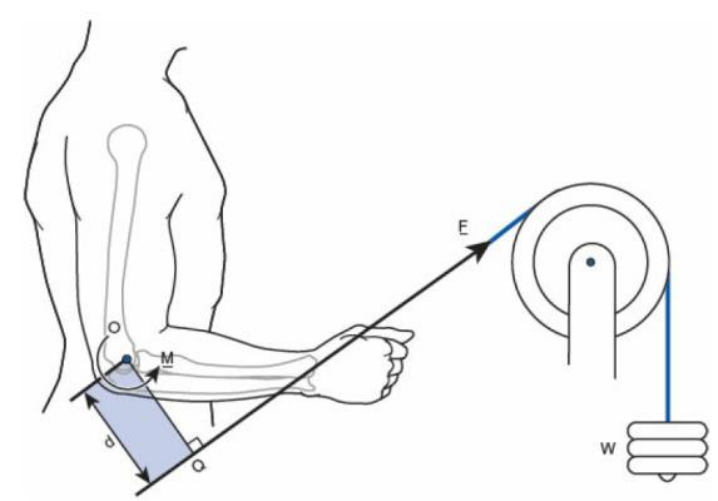

Figura. 12. Definición de cargas. 
ISSN: 2600-5859

$E$ es la fuerza aplicada a la mano por el asa del cable unido al peso en el plato de pesaje $W$, es el peso total del brazo inferior que actúa en el centro de gravedad del brazo inferior. (Latash, 2008)

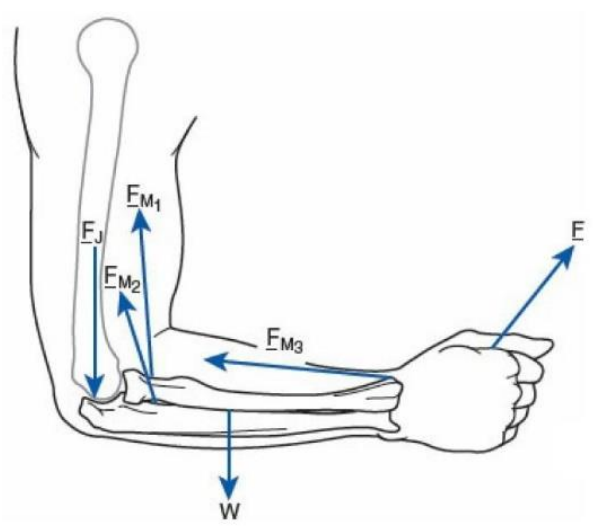

Figura. 13. Diagrama de cuerpo libre de cargas sobre el brazo.

FM1 es la fuerza producida por los bíceps en el radio,

FM3 es la fuerza producida por los músculos braquiorradiales en el radio,

FM2 es la fuerza producida por los músculos braquiales sobre el cúbito, y

FJ es la fuerza de reacción resultante en las articulaciones humeroulnar y humeroradial del codo.

Hay que considerar que las fuerzas de reacción muscular y articular representan los efectos mecánicos de la parte superior del brazo en la parte inferior del brazo. También se debe tener en cuenta que, como se ilustra en la Figura 13 (que no es un diagrama completo de cuerpo libre), las fuerzas de reacción de los músculos y articulaciones opuestas de igual magnitud, también actúan en la parte superior del brazo. Se considera el valor del peso igual a 50N. (Zar Casás, 2016)

\begin{tabular}{lccc}
\hline \multirow{2}{*}{ Músculo } & \multicolumn{3}{c}{ Parámetro } \\
\cline { 2 - 4 } & $F_{0 i}(\mathrm{~N})$ & $A_{i}(\mathrm{~cm} 2)$ & $S_{i}(\%)$ \\
\hline Braquial & 987,3 & 7,1 & $61 \%$ \\
Braquiorradial & 261,3 & 1,9 & $61 \%$ \\
Bíceps largo & 624,3 & 4,5 & $61 \%$ \\
Bíceps corto & 435,6 & 3,1 & $61 \%$ \\
Tríceps medio & 624,3 & 4,5 & $65 \%$ \\
Tríceps lateral & 624,3 & 4,5 & $65 \%$ \\
Tríceps largo & 798,5 & 5,7 & $65 \%$ \\
\hline
\end{tabular}

Figura.14. Cargas de reacción de los músculos 
Generación del modelo físico de acuerdo a las cargas de reacción y condiciones de frontera como son las restricciones de movimientos. (Alawadhi, 2015)

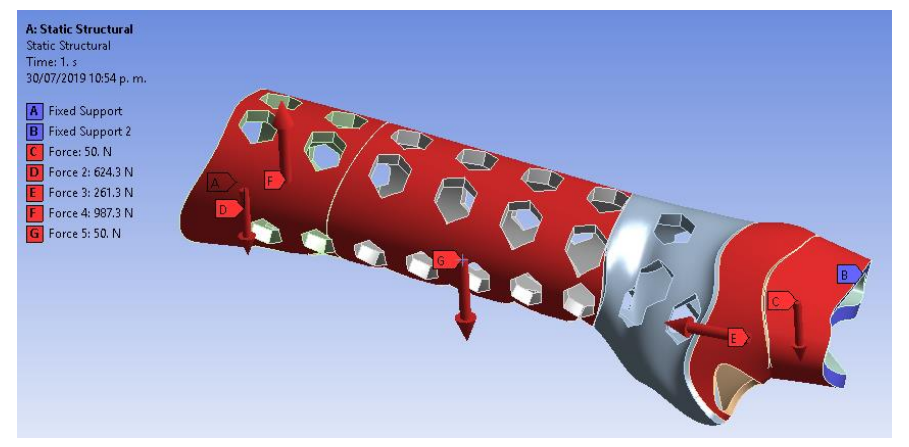

Figura. 15. Asignación de cargas y restricciones al modelo de férula

\section{Proceso de simulación}

El ordenador genera el proceso de cálculo en base a las ecuaciones de energía de distorsión que son parte de resolución del software Ansys Mechanical: en el proceso se muestra graficas en las que se puede analizar la convergencia de la solución en función al cambio de malla vs deformación o esfuerzo de Vonn Misses. Uno de los factores importantes es el número de iteraciones que permitirá conducir a una mayor confiablidad de los resultados.

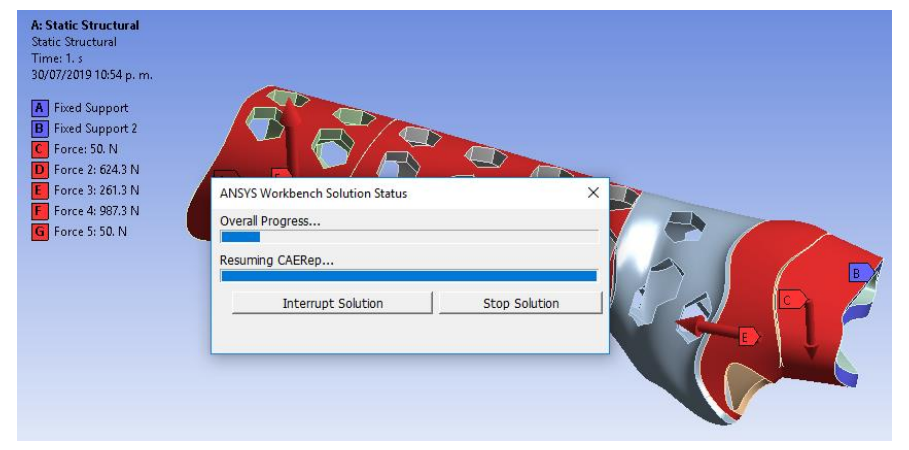

Figura 16. Resolución matemático

\section{Post proceso}

Ansys Mechanical genera como resultado de su simulación el esfuerzo que se produce por la acción de las fuerzas antes mencionadas al igual que las deformaciones en las diferentes direcciones del modelo lo que permite observar el comportamiento de la férula cuando realice su función., permitiendo analizar el funcionamiento de la férula sin necesidad de la construcción. Sin embargo, estos resultados deben ser validados de ser posible con un modelo físico y ensayos en laboratorio de resistencia de materiales. 


\section{Resultados}

Se procede a la generación de la nube de puntos, en este paso para la generación de la férula consiste en el escaneo de miembros superiores de sujetos de pruebas, teniendo en cuenta las opciones del software VxElements de cuerpos semirrígidos y puntos de referencia utilizando los parámetros recomendados. En la siguiente figura se puede observar el comportamiento del escaneo sobre el cuerpo humano y la reconstrucción de los mismos donde se usa el protocolo de Newington para el caso de puntos de referencia.

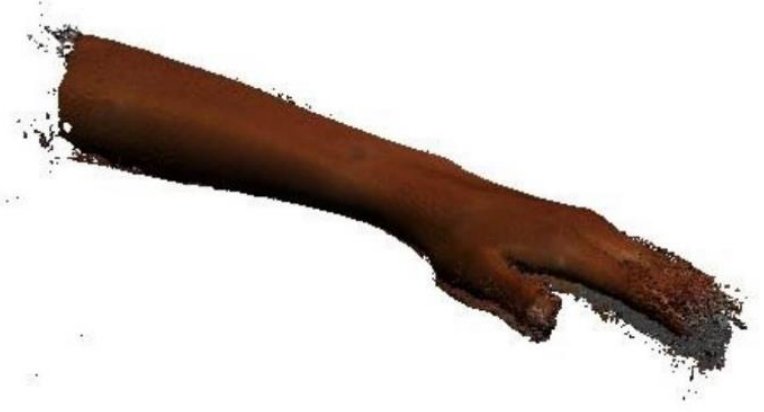

Figura 17. Nube de puntos bajo el Protocolo de Newington.

El siguiente paso es establecer la geometría de escaneado de todos los sujetos de prueba optimizando el mallado correspondiente y quitando los puntos innecesarios para la aplicación mediante el uso del software Geomagic Designer X. Se utiliza una malla provisional, éste es un parámetro importante controlable donde inicialmente se selecciona un valor de $0,55 \mathrm{~mm}$, recomendable, debido a que con valores inferiores los resultados no son favorables, posteriormente se aplica la herramienta "Healing Wizard", la opción permite suavizar la nube de puntos hasta su completa compactación corrigiendo los posibles errores en la malla. Debido a que el cuerpo humano es diferente a todos los materiales utilizables en el mercado, este software presenta una opción Organic, en la misma se pueden observar las siguientes ilustraciones y el acabado de malla que ésta presenta:

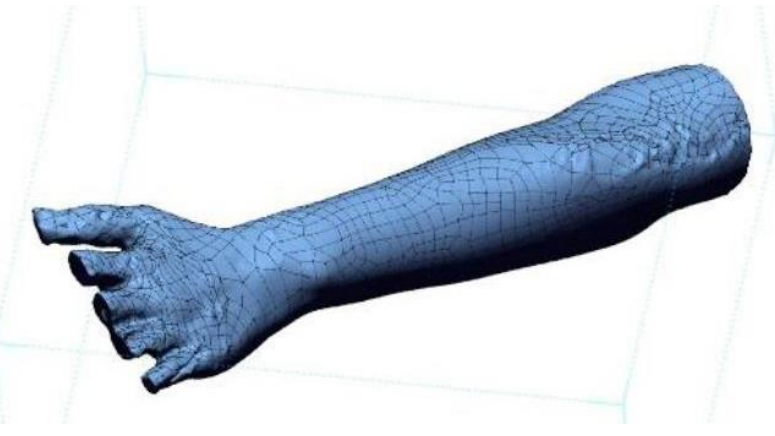

Figura 18. Mallado del sujeto de prueba 
El diseño y modelado de las férulas para los objetivos planteados se lo puede designar de personalizados por los sujetos de prueba, éstos presentan parámetros variables y es necesario considerar todas las necesidades que presenten. La malla exportada en SolidWorks nos permite modelar de mejor manera debido a las características que presentan los cuerpos humanos uniendo segmentos con las coordenadas que el software proporciona.

Para la investigación primeramente se han diseñado férulas ortopédicas infantiles para niños y una mujer adulta. La geometría del miembro superior para estos casos es variable y se debe tomar en cuenta todos los rasgos físicos para que el dispositivo cumpla con la función requerida y se obtengan resultados favorables, tal y como se ilustra en las siguientes imágenes.

La tendencia del uso de la tecnología para el diseño de piezas complejas ha venido evolucionando, de tal manera que ha facilitado y permitido realizar replicas perfectas con el uso del escáner 3D.

El escaneado 3D del miembro superior de una persona ha proporcionado un ahorro de tiempo en la obtención de su geometría, pero también presenta limitaciones por los movimientos involuntarios del paciente durante el escaneado, sin embargo, con la ayuda del software (Geomagic Design) de tratamiento de nube de puntos se ha reconstruido estas zonas defectuosas con bajos márgenes de error.

Se puede interpretar que cuándo se obtiene una nube de puntos es densa en el proceso de escaneado, permite la construcción de la geometría del objeto en un menor tiempo.

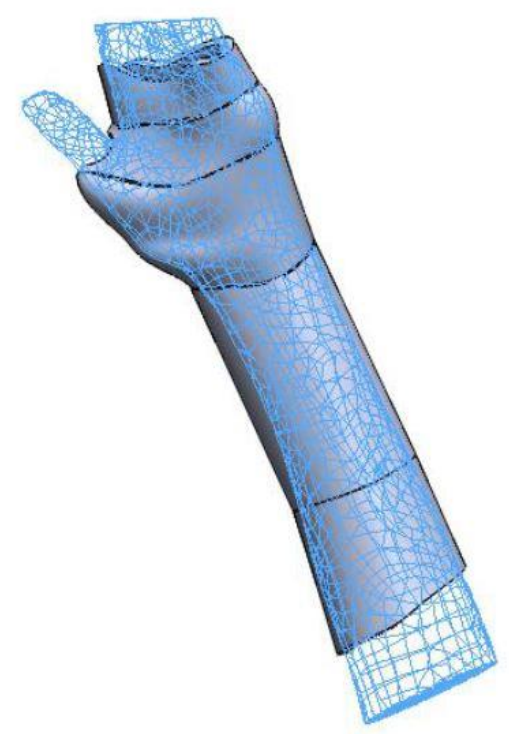

Figura 19. Mallado del sujeto de prueba

La simulación permite obtener resultados del comportamiento estructural mecánico de la férula, en la figura 20 se muestra el comportamiento de deformación cuando actúa sobre la férula las 
ISSN: 2600-5859

cargas descritas anteriormente, teniendo como resultado una deformación máxima de $0.02 \mathrm{~mm}$. La deformación mide el cambio del tamaño o de la forma de la férula debido a los esfuerzos externos producidos por las fuerzas aplicadas sobre la misma, este valor nos permitirá evaluar en parte la ergonomía en el instante de estar colocada en el paciente. Según los resultados la mayor deformación de la férula se presenta en la posición de la mano. (Alawadhi, 2015)

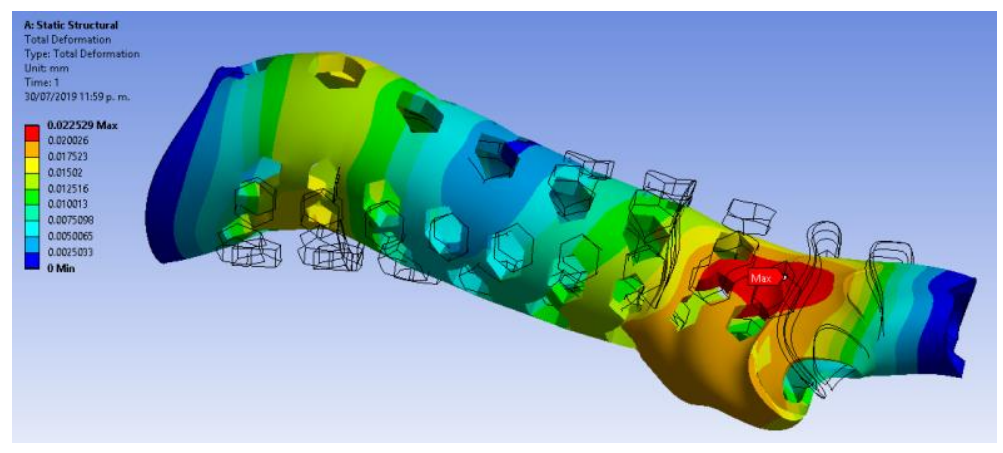

Figura 20. Deformación de la férula

Otro valor que se obtiene es el esfuerzo de von-mises que se conoce como una teoría de fallo elástico que permite determinar los esfuerzos estáticos permisibles además se considera una magnitud física proporcional a la energía de distorsión, en este caso de la férula que nos permite comprobar con el esfuerzo admisible del material utilizado, siendo un esfuerzo máximo de 2.95 $\mathrm{Mpa}$, eso se aprecia en una gama de colores con los valores de los esfuerzos resultantes, como se indica en la figura 21

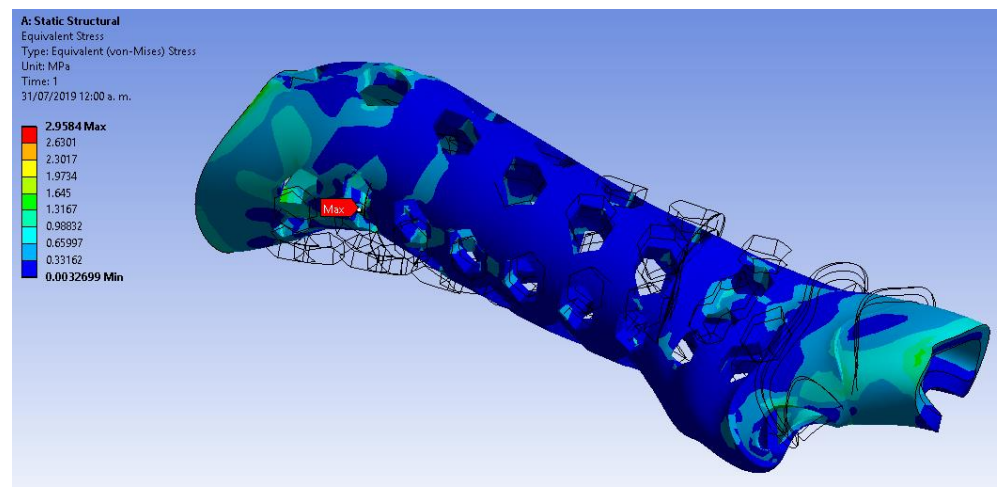

Figura 21. Esfuerzo de Von-Mises de la férula

Mantenemos un criterio de factor se seguridad para validar la resistencia teniendo en los resultados valores mayores a 15, debido a que los esfuerzos generados por la acción de las fuerzas son menores al esfuerzo admisible de material que para el PLA es de $60 \mathrm{MPa}$, lo que indica que la férula se encuentra sobre diseñada para un análisis estático, como se muestra en la figura 22. 


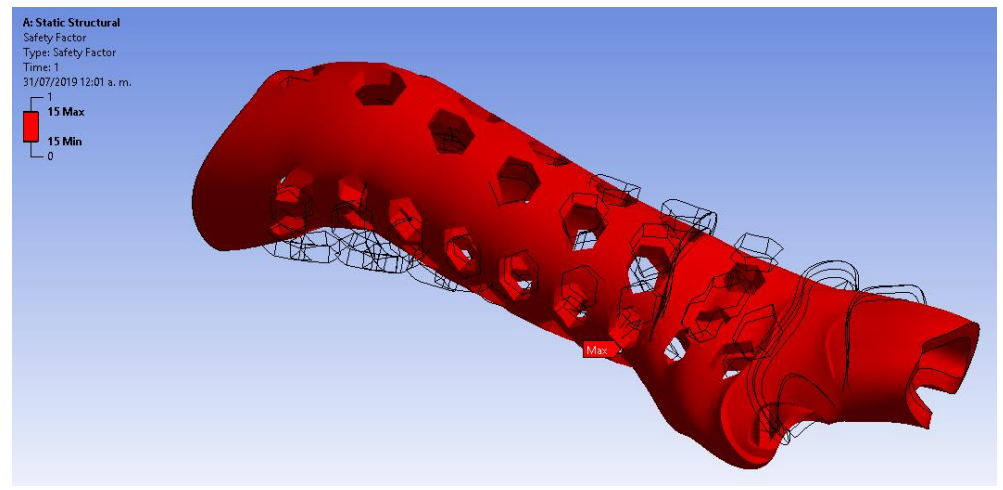

Figura 22. Factor de seguridad de la férula.

Con los resultados antes obtenidos se puede considerar que la férula no sufrirá deformaciones permanentes y no llegará al inicio de la fractura.

En la generación de la geometría y de mallado se debe considerar la forma compleja que tiene la férula en el momento de discretizar el medio físico, por lo que se debe aprovechar los tipos de elementos que se generan en la malla de Ansys como son los elementos tetraedro y hexaedros. Para el efecto del análisis se considera elementos tetraédricos de 10 nodos, obteniéndose mediante la métrica de element quality una malla promedio de 0.711 de una cantidad de 22681 elementos.

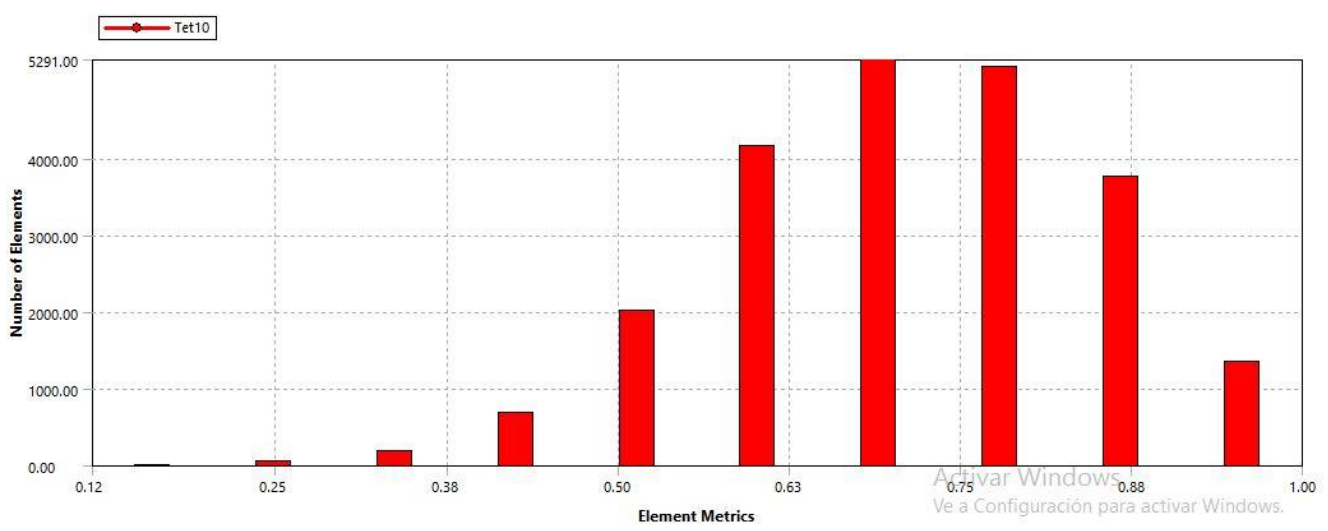

Figura 23. Calidad de malla.

Para manufacturar el diseño de la férula completa se genera la geometría con la extensión. STL, de acuerdo a los protocolos de intercambio de archivos la extensión STL lee el software de impresión 3D en material PLA. 
Las configuraciones para su impresión en 3D se utiliza los parámetros de la tabla 2.

\begin{tabular}{ll}
\hline Altura de la capa en cada pasada & $0,2 \mathrm{~mm}$ \\
\hline Cantidad de relleno de impresión & $30 \%$ \\
Grosor de pared & 0,8 \\
Temperatura del extrusor & $230^{\circ} \mathrm{C}$ \\
Temperatura de la cama & $60^{\circ} \mathrm{C}$ \\
Velocidad de impresión & $60 \%$ \\
\hline
\end{tabular}

Tabla 2.- Parámetros de la impresora 3D

Con los parámetros colocados parra el proceso de impresión se obtiene un archivo GCODE; este código presenta las coordenadas que la impresora debe seguir para así aportar material e ir construyendo la pieza. (León \& Marcos-Fernández, 2019)

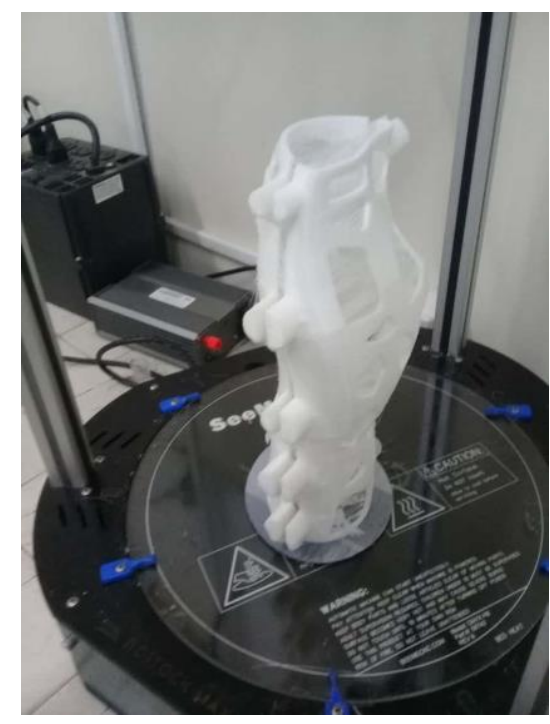

Figura 24. Archivo físico impreso en 3D.

Con los resultados obtenidos de la aplicación de la ingeniería inversa para obtener el modelo geométrico conjuntamente con el análisis computacional de esfuerzos permite la fabricación de la férula mediante la fabricación aditiva utilizando como material un polímero PLA. 
ISSN: 2600-5859

\section{Conclusiones.}

- Estudio de estado del arte de las férulas nos permite evaluar la importancia de la aplicación de la férula en el ámbito de la rehabilitación, generando una personalización de la férula al paciente siendo ergonómica y funcional.

- La obtención del modelo geométrico de la férula a partir de ingeniería inversa genera ventaja para la personalización de la férula en los pacientes.

- Las cargas con las que se presenta el análisis computacional son bibliográficas que sebe establecer los valores reales en función de la edad, peso corporal, genero debido a que las carteristas biomecánicas van a cambiar y los resultados requeridos variaran.

- De acuerdo al factor de seguridad estático se evidencia sobre diseño de la férula lo que se requiere utilizar una herramienta de optimización topológica parta mejorar la geometría con relación a la forma y peso.

- La generación del mallado es importante que se evalué con alguna métrica existente, en el estudio se utilizó un criterio de calidad del elemento denominado element quality donde se obtuvo un valor de 0,711 siendo 1 el valor óptimo, para garantizar la calidad de los resultados.

- Es necesario obtener la curva S-N esfuerzo deformación del material para poder calcular de forma dinámica el factor se seguridad.

\section{Referencias bibliográficas.}

Alawadhi, E. M. (2015). Finite element simulations using ANSYS. CRC Press.

Chang, K.-H. (2014). Product design modeling using CAD/CAE: the computer aided engineering design series. Academic Press.

Chen, Z. (2005). Finite element methods and their applications. Springer Science \& Business Media.

Latash, M. L. (2008). Neurophysiological basis of movement. Human Kinetics.

Lee, H.-H. (2018). Finite Element Simulations with ANSYS Workbench 18. SDC publications.

Nordin, M., \& Frankel, V. H. (2001). Basic biomechanics of the musculoskeletal system. Lippincott Williams \& Wilkins.

Sin, L. T. (2012). Polylactic acid: PLA biopolymer technology and applications. William Andrew.

Zar Casás, A. (2016). Estimación de fuerzas musculares durante el movimiento del brazo humano mediante optimización estático-fisiológica.

León, M., \& Marcos-Fernández, Á. (2019). Impresión 3D con materiales elástoméricos. REVISTA DE PLÁSTICOS MODERNOS, 118(747). 
Molina Osejos, J. V. (2016). Caracterización de materiales termoplásticos de ABS y PLA semirigido impresos en 3D con cinco mallados internos diferentes. Quito, 2016.

Arce GC. Ortesis de miembros superiores. Clasif funciones, prototipos, Caracter Indicaciones [Internet] Lima, Perú Med Rehabil. 2005. 
ISSN: 2600-5859

Vol. 3, $\mathrm{N}^{\circ} 1.2$, p. 137-156, marzo, 2020

\section{PARA CITAR EL ARTÍCULO INDEXADO.}

Pozo Safla, E. R., Aquino Arroba, S. M., Novillo Andrade, G. G., \& Castelo Guevara, E. A. (2020). Simulación de esfuerzos mecánicos sobre las férulas para miembros superiores. ConcienciaDigital, 3(1.2), 137-156. https://doi.org/10.33262/concienciadigital.v3i1.2.1202

\section{【 Ciencia}

El artículo que se publica es de exclusiva responsabilidad de los autores y no necesariamente reflejan el pensamiento de la Revista Ciencia Digital.

El artículo queda en propiedad de la revista y, por tanto, su publicación parcial y/o total en otro medio tiene que ser autorizado por el director de la Revista Ciencia Digital.
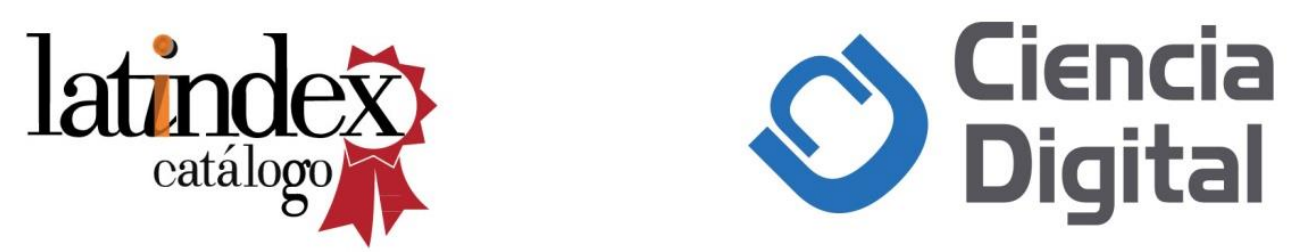